\title{
Treatments for invasive carcinoma of the cervix: what are their impacts on the pelvic floor functions?
}

Alessandra Ferreira de Noronha, Elyonara Mello de Figueiredo, Telma Maria Rossi de Figueiredo Franco, Eduardo Batista Cândido, Agnaldo L. Silva-Filho

Department of Obstetrics, Gynecology and Mastology, Botucatu Medical School State University of São Paulo (UNESP) (AFN, ALSFilho), Botucatu, SP; Department of Physical Therapy, Federal University of Minas Gerais (UFMG) (ELF), Belo Horizonte, Gynecologic Oncologic Unit, Mario Penna Hospital (TMRFF), Belo Horizonte, MG and Department of Obstetrics and Gynecology, Federal University of Minas Gerais (UFMG) (EBC, ALSFilho), Belo Horizonte, MG, Brazil

\section{ABSTRACT}

Aims: Describe the impact of surgery, radiotherapy and chemoradiation in the pelvic floor functions in cervical cancer patients.

Materials and Methods: A prospective study with women submitted to radical hysterectomy $(\mathrm{RH})(\mathrm{n}=20)$, exclusive radiotherapy $(\mathrm{RT})(\mathrm{n}=20)$ or chemoradiation $(\mathrm{CT} / \mathrm{RT})(\mathrm{n}=$ 20) for invasive cervical cancer. Urinary, intestinal and sexual function, as well as vaginal length and pelvic floor muscle contraction were evaluated. Comparisons between groups were performed by Kruskal-Wallis and Chi-square tests $(\mathrm{p}<0.05)$.

Results: The groups were similar in stress urinary incontinence incidence $(p=0.56)$, urinary urgency $(p=0.44)$, urge incontinence $(p=0.54)$, nocturia $(p=0.53)$, incomplete bowel emptying $(p=0.76)$, bowel urgency $(p=0.12)$ and soilage $(p=0.43)$. The CT/ RT group presented a higher urinary frequency $(p<0.001)$ and diarrhea $(p=0.025)$. Patients in the RH group were more sexually active $(p=0.01)$ and experienced less dyspareunia $(p=0.021)$. Vaginal length was shorter in RT group $(5.5 \pm 1.9 \mathrm{~cm})$ and CT/ RT $(5.3 \pm 1.5 \mathrm{~cm})$ than in the RH group $(7.4 \pm 1.1 \mathrm{~cm})(\mathrm{p}<0.001)$. Pelvic floor muscle contraction was similar ( $\mathrm{p}=0.302)$.

Conclusions: RT and CT/RT treatment for cervical carcinoma are more associated to sexual and intestinal dysfunctions.

\section{ARTICLE INFO}

\section{Key words:}

Uterine Cervical Neoplasms; Hysterectomy; Radiation; Chemoradiotherapy;

Pelvic Floor

Int Braz J Urol. 2013; 39: 46-54

Submitted for publication:

February 28, 2012

Accepted after revision:

August 30, 2012

\section{INTRODUCTION}

Cancer of the cervix is the second most common cancer among women worldwide, with an estimated 493,000 new cases and 274,000 deaths (1). Being the most common gynaecological cancer in the developing world, it accounts for two-thirds of cases and continues to be a serious health problem (1). The introduction of screening programs has resulted in the earlier detection of the disease (2) leading to the majority of cervical carcinoma patients diagnosed at a relatively young age. Most of them live for many years with sequelae of the disease and its treatment (3).

The primary treatment for patients with cervical cancer depends on a variety of factors, including staging, tumor characteristics, comorbid medical conditions, and patient and provider preference (4). Radical hysterectomy (RH) and radiotherapy (RT) are effective treatment options for 
patients with cervical cancer (5). The addition of chemotherapy to radiotherapy (chemoradiation $\mathrm{CT} / \mathrm{RT}$ ) results in an absolute improvement in overall survival and a significant reduction in local recurrence for all stages of disease (6-8).

Traditionally, oncologists have focused their efforts on maximizing the overall survival rate of their patients (4). However, when different treatment options result in equivalent clinical outcomes, quality of life (QoL) considerations become particularly important (4). In the management of cervical cancer, attention has turned to reducing morbidity, especially those related to the pelvic organs (2). Pelvic floor dysfunction describes a range of impairments to one or more of the pelvic floor compartments, i.e., the urinary, anorectal, and genital systems as well as to the pelvic floor muscles (2). Autonomic nerve damage during surgery is thought to play a crucial role in the etiology of the pelvic floor dysfunctions that can be seen after RH (9). RT is associated to fibroelastic and muscle alterations (10-12). Moreover, RT and CT can cause ovary failure and early menopause, leading to a decrease in vaginal lubrication and elasticity as well as on the strength of the pelvic floor muscles (13). Although CT/RT treatments increase the chances of some serious acute toxicity, particularly hematologic and gastrointestinal, few trials measured late toxicity, and reported QoL outcomes $(6,14)$. The investigation of the impact of different types of treatment for cervical cancer on the pelvic floor function and QoL of women, might offer relevant information to support oncologists' decision about the best intervention for the patient in question. Therefore, a prospective study was designed to describe the occurrence of pelvic floor dysfunction in women treated for cervical cancer as well as to investigate the impact of the type of treatment on the occurrence of pelvic floor dysfunctions in women who survived cervical cancer.

\section{MATERIALS AND METHODS}

Cancer of the cervix is the second most common cancer among women worldwide, with an estimated 493,000 new cases and 274,000 deaths (1). Being the most common gynaecological cancer in the developing world, it accounts for two-thirds of cases and continues to be a serious health problem (1). The introduction of screening programs has resulted in the earlier detection of the disease (2) leading to the majority of cervical carcinoma patients diagnosed at a relatively young age. Most of them live for many years with sequelae of the disease and its treatment (3).

The primary treatment for patients with cervical cancer depends on a variety of factors, including staging, tumor characteristics, comorbid medical conditions, and patient and provider preference (4). Radical hysterectomy (RH) and radiotherapy (RT) are effective treatment options for patients with cervical cancer (5). The addition of chemotherapy to radiotherapy (chemoradiation $\mathrm{CT} / \mathrm{RT}$ ) results in an absolute improvement in overall survival and a significant reduction in local recurrence for all stages of disease (6-8).

Traditionally, oncologists have focused their efforts on maximizing the overall survival rate of their patients (4). However, when different treatment options result in equivalent clinical outcomes, quality of life (QoL) considerations become particularly important (4). In the management of cervical cancer, attention has turned to reducing morbidity, especially those related to the pelvic organs (2). Pelvic floor dysfunction describes a range of impairments to one or more of the pelvic floor compartments, i.e., the urinary, anorectal, and genital systems as well as to the pelvic floor muscles (2). Autonomic nerve damage during surgery is thought to play a crucial role in the etiology of the pelvic floor dysfunctions that can be seen after RH (9). RT is associated to fibroelastic and muscle alterations (10-12). Moreover, RT and CT can cause ovary failure and early menopause, leading to a decrease in vaginal lubrication and elasticity as well as on the strength of the pelvic floor muscles (13). Although CT/ RT treatments increase the chances of some serious acute toxicity, particularly hematologic and gastrointestinal, few trials measured late toxicity, and reported QoL outcomes $(6,14)$. The investigation of the impact of different types of treatment for cervical cancer on the pelvic floor function and QoL of women, might offer relevant information to support oncologists' decision about the best intervention for the patient in question. The- 
refore, a prospective study was designed to describe the occurrence of pelvic floor dysfunction in women treated for cervical cancer as well as to investigate the impact of the type of treatment on the occurrence of pelvic floor dysfunctions in women who survived cervical cancer.

\section{Statistical analysis}

The data was prospectively collected, digitalized and saved in the SPSS program for Windows version 16.0 (SPSS Inc., Chicago, IL, USA). Comparisons between groups were performed using the Kruskal-Wallis and the Chi-square tests. Post hoc analysis was performed using the Tukey test. The level of significance was set at $p<0.05$. The sample size of 20 participants per group was defined as sufficient to avoid a difference superior to $40 \%$ among the proportions, and to distinguish the standard deviations from the differences in the means among the groups, with a statistical power of $80 \%$ and a confidence level of 95\%.

\section{RESULTS}

The clinical stage of the tumor was I in 25 cases (41.7\%), II in 12 (20\%), III in 22 (36.7\%) and IV in one case (1.7\%). The predominant histological type of carcinoma was squamous cell carcinoma (88.3\%), followed by adenocarcinoma (8.3\%), adenosquamous (1.7\%) and others (1.7\%). There was no difference between groups regarding age, number of pregnancies, menopause status, body mass index and histological type of the tumor. There were differences among groups regarding the tumor clinical stage $(p<0.001)$, (Table-1).

The occurrence of stress urinary incontinency $(p=0.563)$, mictional urgency $(p=0.449)$, urge incontinency $(\mathrm{p}=0.549)$, mictional frequency $(p=0.256)$, vesical pain $(p=0.61)$, recurrent urinary infection $(\mathrm{p}=0.549)$ and nocturia $(\mathrm{p}=0.535)$ was similar between groups (Table-2). There was also no difference regarding pre- and post-treatment subjective perception of the urinary function $(\mathrm{p}=0.308)$ (Figure-1A).

Table 1 - Patient's clinical characteristics according to treatment type.

\begin{tabular}{|c|c|c|c|c|}
\hline & $\begin{array}{c}\mathrm{RH} \\
(n=20)\end{array}$ & $\begin{array}{c}\text { RT } \\
(n=20)\end{array}$ & $\begin{array}{c}\text { CT/RT } \\
(n=20)\end{array}$ & $p$ \\
\hline Age - years mean (SD) & $50.3( \pm 6.4)$ & $52.9( \pm 7.2)$ & $51.5( \pm 7.6)$ & 0.117 \\
\hline Parity mean (SD) & $4.9( \pm 3.0)$ & $4.8( \pm 4.0)$ & $5.2( \pm 3.0)$ & 0.603 \\
\hline Menopause status number (proportion) & $17(85 \%)$ & $18(90 \%)$ & $19(95 \%)$ & 0.908 \\
\hline Hormonal therapy number (proportion) & $1(5 \%)$ & $1(5 \%)$ & 0 & 0.986 \\
\hline $\mathrm{BMI}\left(\mathrm{Kg} / \mathrm{m}^{2}\right)$ mean $(\mathrm{SD})$ & $26.3( \pm 4.6)$ & $26.5( \pm 4.4)$ & $25.8( \pm 3.5)$ & 0.925 \\
\hline Stage (FIGO) & & & & $<0.001$ \\
\hline I & $20(100 \%)$ & $5(25 \%)$ & 0 & \\
\hline II & 0 & $9(45 \%)$ & $3(15 \%)$ & \\
\hline III & 0 & $6(30 \%)$ & $16(80 \%)$ & \\
\hline IV & 0 & 0 & $1(5 \%)$ & \\
\hline
\end{tabular}

Note: $\mathbf{R H}=$ Radical Hysterectomy; $\mathbf{R T}$ = Radiotherapy; $\mathbf{C T} / \mathbf{R T}$ = Chemoradiation. Groups' comparison performed using the Kruskal-Wallis and the Chisquare tests. 
Table 2 - Urinary symptoms according to treatment type.

\begin{tabular}{|c|c|c|c|c|}
\hline & $\mathrm{RH}(\mathrm{n}=20)$ & $\mathrm{RT}(\mathrm{n}=20)$ & $C T / R T(n=20)$ & $p$ \\
\hline Stress incontinence & $6(30 \%)$ & $9(45 \%)$ & $6(30 \%)$ & 0.563 \\
\hline Urgency & $6(30 \%)$ & $12(60 \%)$ & $10(50 \%)$ & 0.449 \\
\hline Urge-incontinence & $9(45 \%)$ & $12(60 \%)$ & $9(45 \%)$ & 0.549 \\
\hline Frequency & $5(25 \%)$ & $10(50 \%)$ & $7(35 \%)$ & 0.256 \\
\hline Bladder pain & $5(25 \%)$ & $10(50 \%)$ & $7(35 \%)$ & 0.61 \\
\hline UTI & $6(30 \%)$ & $4(20 \%)$ & $7(35 \%)$ & 0.549 \\
\hline Nocturia & $6(30 \%)$ & $6(40 \%)$ & $12(60 \%)$ & 0.535 \\
\hline
\end{tabular}

Note: $\mathbf{R H}=$ Radical Hysterectomy; $\mathbf{R T}=$ Radiotherapy; $\mathbf{C T} / \mathbf{R T}=$ Chemoradiation. Groups' comparison performed using the Chi-square test.

There were significant differences between the treatment types regarding the number of daily evacuations $(p=0.001)$ and diarrhea episodes $(\mathrm{p}=0.025)$. CT/RT treatment was positively correlated to frequency of evacuations and diarrhea episodes compared to others groups of treatments. Diarrhea was also significantly more frequent in the RT group when compared to $\mathrm{RH}$ and $\mathrm{CT} / \mathrm{RT}$ (Table-3). There was no differences among groups concerning the sensation of incomplete bladder emptying $(p=0.766)$, evacuation urgency $(p=$ 0.122 ) and soiling ( $p=0.435)$, also in pre- and post- treatment subjective perception of the intestinal function $(\mathrm{p}=0.124)$ (Figure-1B).

Active sex life was more frequent among women submitted to $\mathrm{RH}(90 \%)$ than in women submitted to RT (50\%) and CT/RT (50\%) ( $p=0.01)$. Additionally, a lower dyspareunia rate was observed in the RH group in comparison to the other two groups $(\mathrm{p}=0.021)$ (Table-4). No differences were found among groups regarding a pleasant feeling during sexual intercourse, excitement, orgasm, passion, satisfaction, or number of weekly sexual relations. There was no difference between groups in pre- and post-treatment subjective evaluation of the sexual function $(\mathrm{p}=0.308)$ (Figure-1C).

The vaginal length was shorter in patients submitted to CT/RT $(5.3 \pm 1.5 \mathrm{~cm})$ or RT $(5.5 \pm 1.9$ $\mathrm{cm})$ when compared to the RH group $(7.4 \pm 1.1$ $\mathrm{cm})(\mathrm{p}<0.001)$ (Figure-2).
No differences were observed regarding the contraction capacity of the pelvic floor muscles between RH $(2.9 \pm 1.2)$, RT $(2.2 \pm 1.6)$ or CT/ RT $(2.5 \pm 1.6)(p=0.302)$ patients (Figure-3).

\section{DISCUSSION}

The effects of different types of treatment for cervical carcinoma on the women's pelvic floor functions have recently received some attention. However studies are still scarce and heterogeneous regarding research questions and methods. Despite the trends towards multimodal treatment regimes, to our knowledge, there are no clinical studies that compare the side effects on the pelvic floor functions between patients treated with RH, RT or CT/ $\mathrm{RT}$ for cervical cancer. The present study prospectively evaluated cervical cancer survivors and identified association between types of treatment and pelvic floor dysfunctions. The impact on the pelvic floor functions seems to depend more on the type of treatment than on the stage of the tumor.

In conjunction, the occurrence of urinary dysfunction in the investigated sample was not different from women who did not undergo treatment for cervical cancer. The International Continence Society reports prevalence between 25\% and $45 \%$, indicating that the treatment types investigated in the present study did not contribute to the increase of occurrence of symptoms of uri- 
Figure 1 - Distribution of patients subjective perception of urinary (A), intestinal (B) and sexual (C) functions before and after cervical cancer treatment.
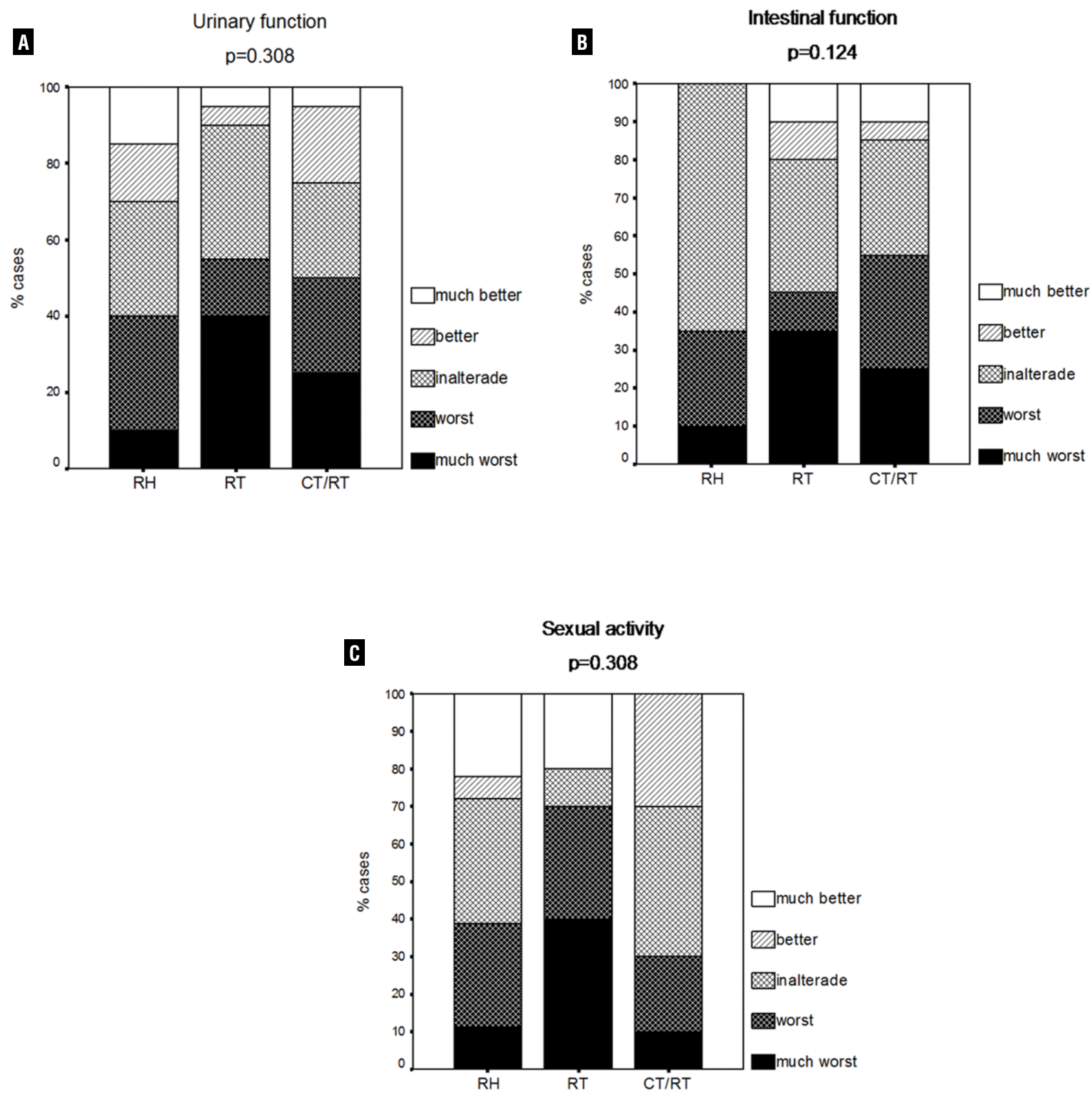

Note: $\mathbf{R H}=$ Radical Hysterectomy; $\mathbf{R T}$ = Radiotherapy; $\mathbf{C T} / \mathbf{R T}$ = Chemo radiation. Groups comparison performed using the Chi-square test. 
Table 3 - Intestinal symptoms according to treatment type.

\begin{tabular}{lcccc}
\hline & $\mathrm{RH}(\mathrm{n}=20)$ & $\mathrm{RT}(\mathrm{n}=20)$ & $\mathrm{QT} / \mathrm{RT}(\mathrm{n}=20)$ & $\mathrm{p}$ \\
\hline Sensation of incomplete bladder emptying & $9(45 \%)$ & $9(45 \%)$ & $11(55 \%)$ & 0.766 \\
Evacuatory urgency & 0 & $3(15 \%)$ & $4(20 \%)$ & 0.122 \\
Diarrhea & $2(10 \%)$ & $9(45 \%)$ & $9(45 \%)$ & 0.025 \\
Fecal soilling & $6(40 \%)$ & $6(30 \%)$ & $10(50 \%)$ & 0.435 \\
Evacuation episodes/day & $0.85 \pm 0.15$ & $1.5 \pm 0.3$ & $2.1 \pm 0.2$ & 0.001 \\
\hline
\end{tabular}

Note: $\mathbf{R H}=$ Radical Hysterectomy; $\mathbf{R T}=$ Radiotherapy; $\mathbf{C T} / \mathbf{R T}=$ Chemoradiation. Groups' comparison performed using the Chi-square test.

Table 4 - Sexual symptoms according to treatment type.

\begin{tabular}{lcccc}
\hline & $\mathrm{RH}(\mathrm{n}=20)$ & $\mathrm{RT}(\mathrm{n}=20)$ & $\mathrm{CT} / \mathrm{RT}(\mathrm{n}=20)$ & $\mathrm{p}$ \\
\hline Pleasant & $3.7 \pm 0.4$ & $3.7 \pm 0.6$ & $4.2 \pm 0.5$ & 0.728 \\
Excitement & $3.3 \pm 0.45$ & $3.4 \pm 0.6$ & $4.0 \pm 0.5$ & 0.652 \\
Orgasm & $3.8 \pm 0.5$ & $2.8 \pm 0.5$ & $3.8 \pm 0.6$ & 0.336 \\
Passion & $4.4 \pm 0.4$ & $4.1 \pm 0.7$ & $5.2 \pm 0.5$ & 0.414 \\
Satisfaction & $4.4 \pm 0.5$ & $3.8 \pm 0.6$ & $4.9 \pm 0.5$ & 0.424 \\
Pain & $1.7 \pm 0.3$ & $3.6 \pm 0.7$ & $2.9 \pm 0.5$ & 0.021 \\
Frequency & $1.9 \pm 0.2$ & $1.6 \pm 0.3$ & $1.9 \pm 0.4$ & 0.706 \\
\hline
\end{tabular}

Note: $\mathbf{R H}=$ Radical Hysterectomy; $\mathbf{R T}$ = Radiotherapy; $\mathbf{C T} / \mathbf{R T}=$ Chemo radiation. Groups' comparison performed using the Kruskal-Wallis test.

Figure 2 - Vaginal length according to treatment type.

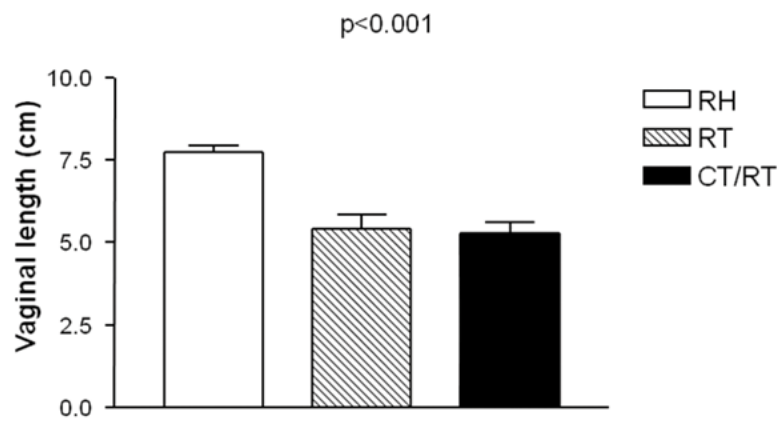

Note: $\mathbf{R H}=$ Radical Hysterectomy; $\mathbf{R T}=$ Radiotherapy; $\mathbf{C T} / \mathbf{R T}=$ Chemo radiation. Groups' comparison performed using the Kruskal-Wallis test. nary dysfunctions (14). The type of treatment did also not influence urinary function. These results are similar to those reported by Lin et al., 1998 who also did not find any differences in detrusor instability or bladder compliance in women submitted to the RH and/or RT, evaluated by urodynamics (19). Further studies involving similar participants should be developed to corroborate these findings.

Abnormal anorectal function occurs frequently following pelvic irradiation for gynecological malignant diseases. In the present study, RT and CT/RT were associated with high rates of diarrhea and frequent evacuations. These results are corroborated by others who have also documented 
Figure 3 - Distribution of pelvic floor muscles contraction (Oxford scale) according to treatment type.

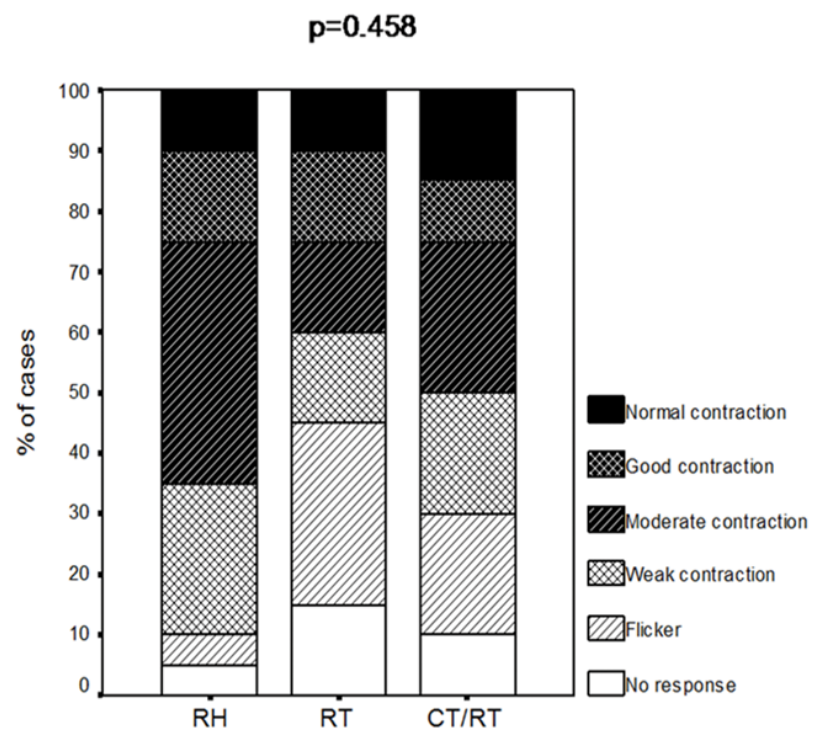

Note: $\mathbf{R H}=$ Radical Hysterectomy; $\mathbf{R T}=$ Radiotherapy; $\mathbf{C T} / \mathbf{R T}=$ Chemo radiation. Groups' comparison performed using the Chi-square test.

the side effects of pelvic radiation on defecation $(20,21)$. This is relevant because some data suggest that acute toxicity and diarrhea during radiation therapy of cervical carcinoma significantly increased the risk of severe late toxicity, therefore, affecting patient's quality of life (22). Bile acid malabsorption and bacterial overgrowth are frequently found in patients with chronic or intermittent diarrhea (23). The reduced rectal reservoir capacity and impaired sensory functions were crucial factors for functional disorder in such patients $(12,24)$. In addition, radiation damage to the external anal sphincter muscle was considered to be an important cause of motor dysfunction $(12,24)$.

Most of the literature concerning sexual functioning among cervical cancer patients has focused on those with early stage disease. Our finding that women who received RT or CT/RT had increased levels of dyspareunia is consistent with earlier studies $(3,25,26)$. The decrease in vaginal length may explain in part the increased dyspareunia. Vaginal shortening can occur during the course of intracavity and external irradiation (27-29). In the present study the vaginal length was smaller in patients submitted to CT/RT or RT when compared to the $\mathrm{RH}$ group. Although the sexual activity rate is lower in RT and CT/RT groups, they are able to enjoy sexual intercourse similar to patients after surgery. The low sexual activity in cancer patients is a limitation regarding the interpretation of sexual function in this study. However, the results are consistent with those previously reported (5).

Pelvic floor muscles dysfunction is correlated with urinary, sexual, or gastroenterological complaints and pelvic organ prolapse $(30,31)$. However, in the present study, no differences were observed regarding the capacity of pelvic floor muscle contraction evaluated through bidigital palpation. This result might be due to the good capacity of the pelvic floor muscles contraction in the sample investigated, since most women presented degrees of contraction higher than or equal to 2 (weak) on the modified Oxford scale.

There was no difference regarding preand post-treatment subjective perception of the urinary, intestinal and sexual functions. The relief resulting from the completion of potentially curative treatment may also have contributed to this result despite changes in pelvic functions.

\section{CONCLUSIONS}

Our data suggest that RT and $\mathrm{CT} / \mathrm{RH}$ are associated to more sexual dysfunctions, as less active sexual life and dyspareunia, and more intestinal dysfunctions, as constipation and also diarrhea, after treatment for invasive cervical carcinoma. Further studies are needed to develop techniques that will minimize the development of sexual and intestinal dysfunction following RT and CT/RT and to improve clinicians' awareness of the prevalence and impact of these symptoms on their patients' functional status and quality of life.

\section{CONFLICT OF INTEREST}

None declared.

\section{REFERENCES}

1. Parkin DM, Bray F, Ferlay J, Pisani P: Global cancer statistics, 2002. CA Cancer J Clin. 2005; 55: 74-108. 
2. Jackson KS, Naik R: Pelvic floor dysfunction and radical hysterectomy. Int J Gynecol Cancer. 2006; 16: 354-63.

3. Park SY, Bae DS, Nam JH, Park CT, Cho CH, Lee JM, et al.: Quality of life and sexual problems in disease-free survivors of cervical cancer compared with the general population. Cancer. 2007; 110: 2716-25.

4. Frumovitz M, Sun CC, Schover LR, Munsell MF, Jhingran A, Wharton JT, et al.: Quality of life and sexual functioning in cervical cancer survivors. Oncol. 2005; 23: 7428-36.

5. Greimel ER, Winter R, Kapp KS, Haas J: Quality of life and sexual functioning after cervical cancer treatment: a long-term follow-up study. Psychooncology. 2009; 18: 476-82.

6. Chemoradiotherapy for Cervical Cancer Meta-Analysis Collaboration: Reducing uncertainties about the effects of chemoradiotherapy for cervical cancer: a systematic review and metaanalysis of individual patient data from 18 randomized trials. J Clin Oncol. 2008; 26: 5802-12.

7. Green J, Kirwan J, Tierney J, Vale C, Symonds P, Fresco L, et al.: Concomitant chemotherapy and radiation therapy for cancer of the uterine cervix. Cochrane Database Syst Rev. 2005; (3):CD002225.

8. Kirwan JM, Symonds P, Green JA, Tierney J, Collingwood M, Williams CJ: A systematic review of acute and late toxicity of concomitant chemoradiation for cervical cancer. Radiother Oncol. 2003; 68: 217-26.

9. Pieterse QD, Maas CP, ter Kuile MM, Lowik M, van Eijkeren MA, Trimbos JB, et al.: An observational longitudinal study to evaluate miction, defecation, and sexual function after radical hysterectomy with pelvic lymphadenectomy for early-stage cervical cancer. Int J Gynecol Cancer. 2006; 16: 1119-29.

10. Da Silva GM, Berho M, Wexner SD, Efron J, Weiss EG, Nogueras $\mathrm{JJ}$, et al.: Histologic analysis of the irradiated anal sphincter. Dis Colon Rectum. 2003; 46: 1492-7.

11. Reis ED, Vine AJ, Heimann T: Radiation damage to the rectum and anus: pathophysiology, clinical features and surgical implications. Colorectal Dis. 2002; 4: 2-12.

12. Kim GE, Lim JJ, Park W, Park HC, Chung EJ, Seong J, et al.: Sensory and motor dysfunction assessed by anorectal manometry in uterine cervical carcinoma patients with radiationinduced late rectal complication. Int J Radiat Oncol Biol Phys. 1998; 41: 835-41.

13. Bruner DW, Nolte SA, Shahin MS, Huang HQ, Sobel E, Gallup $D$, et al.: Measurement of vaginal length: Reliability of the vaginal sound--a Gynecologic Oncology Group study. Int J Gynecol Cancer. 2006; 16: 1749-55.

14. Paul Abrams LC, Saad Khory, Alan Wein: 4th International Consultation on Incontinence. 4th International Consultation on Incontinence. Paris: () Health Publication Ltd. 2009: pp. 1-1821.

15. Kelleher CJ, Cardozo LD, Khullar V, Salvatore S: A new questionnaire to assess the quality of life of urinary incontinent women. Br J Obstet Gynaecol. 1997; 104: 1374-9.
16. Temple LK, Bacik J, Savatta SG, Gottesman L, Paty PB, Weiser MR, et al.: The development of a validated instrument to evaluate bowel function after sphincter-preserving surgery for rectal cancer. Dis Colon Rectum. 2005; 48: 1353-65.

17. Dennerstein L, Lehert P, Dudley E: Short scale to measure female sexuality: adapted from McCoy Female Sexuality Questionnaire. J Sex Marital Ther. 2001; 27: 339-51.

18. Laycock J: Clinical evaluation of the pelvic floor. In: Schuessler B (ed.), Pelvic floor re-education: principles and practice. Springer. 1994: pp. 42-8.

19. Lin HH, Sheu BC, Lo MC, Huang SC: Abnormal urodynamic findings after radical hysterectomy or pelvic irradiation for cervical cancer. Int J Gynaecol Obstet. 1998; 63: 169-74.

20. Iwamoto T, Nakahara S, Mibu R, Hotokezaka M, Nakano $\mathrm{H}$, Tanaka M: Effect of radiotherapy on anorectal function in patients with cervical cancer. Dis Colon Rectum. 1997; 40: 693-7. Erratum in: Dis Colon Rectum 1997; 40: 1298.

21. Hsu WC, Chung NN, Chen YC, Ting LL, Wang PM, Hsieh PC, et al.: Comparison of surgery or radiotherapy on complications and quality of life in patients with the stage IB and IIA uterine cervical cancer. Gynecol Oncol. 2009; 115: 41-5.

22. Wang CJ, Leung SW, Chen HC, Sun LM, Fang FM, Huang EY, et al.: The correlation of acute toxicity and late rectal injury in radiotherapy for cervical carcinoma: evidence suggestive of consequential late effect (CQLE). Int J Radiat Oncol Biol Phys. 1998; 40: 85-91.

23. Danielsson A, Nyhlin H, Persson H, Stendahl U, Stenling R, Suhr 0: Chronic diarrhoea after radiotherapy for gynaecological cancer: occurrence and aetiology. Gut. 1991; 32: 1180-7.

24. Yeoh E, Sun WM, Russo A, Ibanez L, Horowitz M: A retrospective study of the effects of pelvic irradiation for gynecological cancer on anorectal function. Int $\mathrm{J}$ Radiat Oncol Biol Phys. 1996; 35: 1003-10.

25. Schover LR, Fife M, Gershenson DM: Sexual dysfunction and treatment for early stage cervical cancer. Cancer. 1989; 63: 204-12.

26. Jensen PT, Groenvold M, Klee MC, Thranov I, Petersen MA, Machin D: Longitudinal study of sexual function and vaginal changes after radiotherapy for cervical cancer. Int J Radiat Oncol Biol Phys. 2003; 56: 937-49. Erratum in: Int J Radiat Oncol Biol Phys. 2004; 58: 1321.

27. Katz A, Njuguna E, Rakowsky E, Sulkes A, Sulkes J, Fenig E: Early development of vaginal shortening during radiation therapy for endometrial or cervical cancer. Int J Gynecol Cancer. 2001; 11: 234-5.

28. Brand AH, Bull CA, Cakir B: Vaginal stenosis in patients treated with radiotherapy for carcinoma of the cervix. Int $J$ Gynecol Cancer. 2006; 16: 288-93.

29. Bruner DW, Lanciano R, Keegan M, Corn B, Martin E, Hanks GE: Vaginal stenosis and sexual function following intracavitary radiation for the treatment of cervical and endometrial carcinoma. Int J Radiat Oncol Biol Phys. 1993; 27: 825-30. 
30. Voorham-van der Zalm PJ, Lycklama A Nijeholt GA, Elzevier HW, Putter H, Pelger RC: "Diagnostic investigation of the pelvic floor": a helpful tool in the approach in patients with complaints of micturition, defecation, and/or sexual dysfunction. J Sex Med. 2008; 5: 864-71.
31. Ghetti C, Gregory WT, Edwards SR, Otto LN, Clark AL: Severity of pelvic organ prolapse associated with measurements of pelvic floor function. Int Urogynecol J Pelvic Floor Dysfunct. 2005; 16: 432-6.

Correspondence address:

Dr. Agnaldo Lopes da Silva Filho

Department of Obstetrics and Gynecology, Federal University of Minas Gerais (UFMG), Belo Horizonte

Avenida Professor Alfredo Balena 190, Santa Efigênia Belo Horizonte, MG, 30130-100, Brazil

Fax: + 5531 3409-9765

E-mail:agnaldo.ufmg@gmail.com 